\title{
Meir-Keeler type contractions in partially ordered $G$-metric spaces
}

\author{
Hui-Sheng Ding ${ }^{1 *}$ and Erdal Karapınar ${ }^{2}$
}

\section{${ }^{*}$ Correspondence:}

dinghs@mail.ustc.edu.cn

${ }^{1}$ College of Mathematics and

Information Science, Jiangxi Normal

University, Nanchang, Jiangxi

330022, People's Republic of China

Full list of author information is

available at the end of the article

\begin{abstract}
In this paper, we establish several fixed point theorems for Meir-Keeler type contractions in partially ordered $G$-metric spaces.
\end{abstract}

MSC: 46N40; 47H10; 54H25; 46T99

Keywords: Meir-Keeler type contraction; G-metric space; fixed point

\section{Introduction and preliminaries}

There are three main motivations for this paper. The first is the introduction of the concept of a G-metric space and fixed point theorems on G-metric spaces. The second is the works on fixed point theorems of Meir-Keeler type contractions. The third is some recent works on fixed point theorems in a partially ordered set.

In this paper, we will combine these ideas and present some new results. In fact, due to the powerfulness of the classical Banach contraction principle in nonlinear analysis, various generalizations of the classical Banach contraction principle have been of great interest for many authors (see, e.g., [1-26]). Next, let us recall some definitions and known results.

In 2004, Mustafa and Sims [15] introduced the concept of G-metric spaces as follows.

Definition 1 (See [15]) Let $X$ be a non-empty set, $G: X \times X \times X \rightarrow \mathbb{R}^{+}$be a function satisfying the following properties:

(G1) $G(x, y, z)=0$ if $x=y=z$,

(G2) $0<G(x, x, y)$ for all $x, y \in X$ with $x \neq y$,

(G3) $G(x, x, y) \leq G(x, y, z)$ for all $x, y, z \in X$ with $y \neq z$,

(G4) $G(x, y, z)=G(x, z, y)=G(y, z, x)=\cdots$ (symmetry in all three variables),

(G5) $G(x, y, z) \leq G(x, a, a)+G(a, y, z)$ for all $x, y, z, a \in X$ (rectangle inequality).

Then the function $G$ is called a generalized metric or, more specifically, a $G$-metric on $X$, and the pair $(X, G)$ is called a $G$-metric space.

Every $G$-metric on $X$ defines a metric $d_{G}$ on $X$ by

$$
d_{G}(x, y)=G(x, y, y)+G(y, x, x) \quad \text { for all } x, y \in X .
$$


Example 2 Let $(X, d)$ be a metric space. The function $G: X \times X \times X \rightarrow[0,+\infty)$, defined by

$$
G(x, y, z)=\max \{d(x, y), d(y, z), d(z, x)\}
$$

or

$$
G(x, y, z)=d(x, y)+d(y, z)+d(z, x)
$$

for all $x, y, z \in X$, is a $G$-metric on $X$.

Definition 3 (See [15]) Let $(X, G)$ be a $G$-metric space, and let $\left\{x_{n}\right\}$ be a sequence of points of $X$, therefore, we say that $\left(x_{n}\right)$ is $G$-convergent to $x \in X$ if $\lim _{n, m \rightarrow+\infty} G\left(x, x_{n}, x_{m}\right)=0$, that is, for any $\varepsilon>0$, there exists $N \in \mathbb{N}$ such that $G\left(x, x_{n}, x_{m}\right)<\varepsilon$ for all $n, m \geq N$. We call $x$ the limit of the sequence and write $x_{n} \rightarrow x$ or $\lim _{n \rightarrow+\infty} x_{n}=x$.

Proposition 4 (See [15]) Let $(X, G)$ be a G-metric space. The following are equivalent:

(1) $\left\{x_{n}\right\}$ is G-convergent to $x$,

(2) $G\left(x_{n}, x_{n}, x\right) \rightarrow 0$ as $n \rightarrow+\infty$,

(3) $G\left(x_{n}, x, x\right) \rightarrow 0$ as $n \rightarrow+\infty$,

(4) $G\left(x_{n}, x_{m}, x\right) \rightarrow 0$ as $n, m \rightarrow+\infty$.

Definition 5 (See [15]) Let $(X, G)$ be a $G$-metric space. A sequence $\left\{x_{n}\right\}$ is called a $G$ Cauchy sequence if for any $\varepsilon>0$, there is $N \in \mathbb{N}$ such that $G\left(x_{n}, x_{m}, x_{l}\right)<\varepsilon$ for all $m, n, l \geq$ $N$, that is, $G\left(x_{n}, x_{m}, x_{l}\right) \rightarrow 0$ as $n, m, l \rightarrow+\infty$.

Proposition 6 (See [15]) Let $(X, G)$ be a G-metric space. Then the following are equivalent:

(1) the sequence $\left\{x_{n}\right\}$ is G-Cauchy,

(2) for any $\varepsilon>0$, there exists $N \in \mathbb{N}$ such that $G\left(x_{n}, x_{m}, x_{m}\right)<\varepsilon$ for all $m, n \geq N$.

Definition 7 (See [15]) A G-metric space $(X, G)$ is called $G$-complete if every $G$-Cauchy sequence is $G$-convergent in $(X, G)$.

Definition 8 (See [15]) Let $(X, G)$ be a $G$-metric space. A mapping $T: X \rightarrow X$ is said to be $G$-continuous if $\left\{T\left(x_{n}\right)\right\}$ is $G$-convergent to $T(x)$ where $\left\{x_{n}\right\}$ is any $G$-convergent sequence converging to $x$.

Definition 9 Let $(X, \preceq)$ be a partially ordered set, $(X, G)$ be a $G$-metric space. A partially ordered $G$-metric space $(X, G, \preceq)$ is called ordered complete if for each convergent sequence $\left\{x_{n}\right\}_{n=0}^{\infty} \subset X$, the following conditions hold:

$\left(\mathrm{OC}_{1}\right)$ if $\left\{x_{n}\right\}$ is a non-increasing sequence in $X$ such that $x_{n} \rightarrow x^{*}$, then $x^{*} \preceq x_{n} \forall n \in \mathbb{N}$, $\left(\mathrm{OC}_{2}\right)$ if $\left\{y_{n}\right\}$ is a non-decreasing sequence in $X$ such that $y_{n} \rightarrow y^{*}$, then $y^{*} \succeq y_{n} \forall n \in \mathbb{N}$.

In [14], Mustafa characterized the well-known Banach contraction principle mapping in the context of $G$-metric spaces in the following ways. 
Theorem 10 (See [14]) Let $(X, G)$ be a complete G-metric space and $T: X \rightarrow X$ be a mapping satisfying the following condition for all $x, y, z \in X$ :

$$
G(T x, T y, T z) \leq k G(x, y, z)
$$

where $k \in[0,1)$. Then $T$ has a unique fixed point.

Theorem 11 (See [14]) Let $(X, G)$ be a complete G-metric space and $T: X \rightarrow X$ be a mapping satisfying the following condition for all $x, y \in X$ :

$$
G(T x, T y, T y) \leq k G(x, y, y)
$$

where $k \in[0,1)$. Then $T$ has a unique fixed point.

Remark 12 The condition (1.2) implies the condition (1.3). The converse is true only if $k \in\left[0, \frac{1}{2}\right)$. For details, see [14].

Ran and Reurings [22] proved the analog of the Banach contraction mapping principle for continuous self-mappings under certain conditions in the context of a partially ordered set. In this paper [22], the authors solved a matric equation as an application. Following this initial paper, Nieto and López [20] published the paper in which the authors extended the results of Ran and Reurings [22] for a mapping $T$ not necessarily continuous by assuming an additional hypothesis on $(X, \preceq, d)$.

An interesting and general contraction condition for self-maps in metric spaces was considered by Meir and Keeler [13] in 1969.

Definition 13 Let $(X, d)$ be a metric space and $T$ be a self-map on $X$. Then $T$ is called a Meir-Keeler type contraction whenever for each $\varepsilon>0$ there exists $\delta>0$ such that for any $x, y \in X$,

$$
\varepsilon \leq d(x, y)<\varepsilon+\delta \quad \Rightarrow \quad d(T x, T y)<\varepsilon .
$$

Recently, Harjani, Lopez and Sadarangani [7] extended the classical result in [13] to partially ordered metric spaces. In fact, they proved several interesting results for fixed points of Meir-Keeler contractions in a complete metric space endowed with a partial order. For more related results, we refer the reader to $[9,10,25]$ and references therein. Following this line of thought, we introduce a generalized Meir-Keeler type contraction on G-metric spaces and extend the results of $[7,13]$ in the context of partially ordered $G$-metric spaces. We say that the tripled $(x, y, z) \in X^{3}$ is distinct if at least one of the following holds:
(i) $x \neq y$,
(ii) $y \neq z$,
(iii) $x \neq z$

The tripled $(x, y, z) \in X^{3}$ is called strictly distinct if all inequalities (i)-(iii) hold.

Definition 14 Let $(X, G, \preceq)$ be a partially ordered $G$-metric space. Suppose that $T: X \rightarrow X$ is a self-mapping satisfying the following condition: 
For each $\varepsilon>0$, there exists $\delta>0$ such that for any $x, y, z \in X$ with $x \preceq y \preceq z$,

$$
\varepsilon \leq G(x, y, z)<\varepsilon+\delta \Rightarrow G(T x, T y, T z)<\varepsilon .
$$

Then $T$ is called G-Meir-Keeler contractive.

Remark 15 Notice that if $T: X \rightarrow X$ is G-Meir-Keeler contractive on a G-metric space $(X, G)$, then $T$ is contractive, that is,

$$
G(T x, T y, T z)<G(x, y, z)
$$

for all distinct tripled $(x, y, z) \in X^{3}$ with $x \preceq y \preceq z$.

Definition 16 Let $(X, \preceq)$ be a partially ordered set and $T: X \rightarrow X$ be a mapping. We say that $T$ is nondecreasing if for $x, y \in X$,

$$
x \preceq y \text { implies } T x \preceq T y \text {. }
$$

Definition 17 Let $(X, G, \preceq)$ be a $G$-metric space. Suppose that $T: X \rightarrow X$ is a self-mapping satisfying the following condition:

Given $\varepsilon>0$, there exists $\delta>0$ such that for any $x, y \in X$ with $x \preceq y$,

$$
\varepsilon \leq G(x, y, y)<\varepsilon+\delta \Rightarrow G(T x, T y, T y)<\varepsilon .
$$

Then $T$ is called G-Meir-Keeler contractive of second type.

Remark 18 It is easy to see that a G-Meir-Keeler contraction must be G-Meir-Keeler contractive of second type. In addition, if $T: X \rightarrow X$ is G-Meir-Keeler contractive of second type on a partially ordered $G$-metric space $(X, G, \preceq)$, then

$$
G(T x, T y, T y)<G(x, y, y)
$$

for all $(x, y) \in X^{2}$ with $x \prec y$. Moreover, we have

$$
G(T x, T y, T y) \leq G(x, y, y)
$$

for all $(x, y) \in X^{2}$ with $x \preceq y$.

\section{Main results}

In this paper, we discuss the existence of fixed points for a Meir-Keeler type contraction in partially ordered $G$-metric spaces.

Theorem 19 Let $(X, \preceq)$ be a partially ordered set endowed with a G-metric and $T: X \rightarrow X$ be a given mapping. Suppose that the following conditions hold:

(i) $(X, G)$ is G-complete;

(ii) $T$ is nondecreasing (with respect to $\preceq$ );

(iii) there exists $x_{0} \in X$ such that $x_{0} \preceq T x_{0}$; 
(iv) $T$ is G-continuous;

(v) $T: X \rightarrow X$ is G-Meir-Keeler contractive of second type.

Then $T$ has a fixed point. Moreover, if for all $(x, y) \in X \times X$ there exists $w \in X$ such that $x \preceq w$ and $y \preceq w$, we obtain the uniqueness of the fixed point.

Proof The following proof follows the same lines as previous proofs of related results in [7, 13], but we reproduce it for the sake of completeness. More precisely, the first part of the proof for Theorem 19, the proof up to equation (2.13), is analogous to the corresponding proof of Harjani et al. in [7]. But, for the general readership, we give all the details here.

Take $x_{0} \in X$ such that the condition (iii) holds, that is, $x_{0} \preceq T x_{0}$. We construct an iterative sequence $\left\{x_{n}\right\}$ in $X$ as follows:

$$
x_{n}=T x_{n-1} \quad \text { for } n \geq 1 .
$$

Taking into account that $T$ is a non-decreasing mapping together with (2.1), we have $x_{0} \preceq$ $T x_{0}=x_{1}$ implies $x_{1}=T x_{0} \preceq T x_{1}=x_{2}$. By induction, we get

$$
x_{0} \preceq x_{1} \preceq x_{2} \preceq \cdots \preceq x_{n-1} \preceq x_{n} \preceq x_{n+1} \preceq \cdots .
$$

Suppose that there exists $n_{0}$ such that $x_{n_{0}}=x_{n_{0}+1}$. Since $x_{n_{0}}=x_{n_{0}+1}=T x_{n_{0}}$, then $x_{n_{0}}$ is the fixed point of $T$, which completes the existence part of the proof. Suppose that $x_{n} \neq x_{n+1}$ for all $n \in \mathbb{N}$. Thus, by (2.2) we have

$$
x_{0} \prec x_{1} \prec x_{2} \prec \cdots \prec x_{n-1} \prec x_{n} \prec x_{n+1} \prec \cdots
$$

By (G2), we have

$$
G\left(x_{n}, x_{n+1}, x_{n+1}\right)>0
$$

for all $n=0,1,2, \ldots$. By Remark 18 , we observe that for all $n=0,1,2, \ldots$,

$$
G\left(x_{n+1}, x_{n+2}, x_{n+2}\right)=G\left(T x_{n}, T x_{n+1}, T x_{n+1}\right)<G\left(x_{n}, x_{n+1}, x_{n+1}\right) .
$$

Define $t_{n}=G\left(x_{n}, x_{n+1}, x_{n+1}\right)$. Due to (2.5), the sequence $\left\{t_{n}\right\}$ is a (strictly) decreasing sequence in $\mathbb{R}^{+}$and thus it is convergent, say $t \in \mathbb{R}^{+}$. We claim that $t=0$. Suppose, to the contrary, that $t>0$. Thus, we have

$$
0<t<G\left(x_{n}, x_{n+1}, x_{n+1}\right) \quad \text { for all } n=0,1,2, \ldots
$$

Assume $\varepsilon=t>0$. Then by hypothesis, there exists a convenient $\delta(\varepsilon)>0$ such that (1.8) holds. On the other hand, due to the definition of $\varepsilon$, there exists $n_{0} \in \mathbb{N}$ such that

$$
\varepsilon<t_{n_{0}}=G\left(x_{n_{0}}, x_{n_{0}+1}, x_{n_{0}+1}\right)<\varepsilon+\delta .
$$

Taking the condition (1.8) into account, the expression (2.7) yields that

$$
t_{n_{0}+1}=G\left(x_{n_{0}+1}, x_{n_{0}+2}, x_{n_{0}+2}\right)=G\left(T x_{n_{0}}, T x_{n_{0}+1}, T x_{n_{0}+1}\right)<\varepsilon=t,
$$

which contradicts (2.6). Hence $t=0$, that is, $\lim _{n \rightarrow \infty} t_{n}=0$. 
We will show that $\left\{x_{n}\right\}_{n=0}^{\infty}$ is a $G$-Cauchy sequence. $\forall \varepsilon>0$, by the hypothesis, there exists a suitable $\delta(\varepsilon)>0$ such that (1.8) holds. Without loss of generality, we assume $\delta<\varepsilon$. Since $t=0$, there exists $N_{0} \in \mathbb{N}$ such that

$$
t_{n-1}=G\left(x_{n-1}, x_{n}, x_{n}\right)<\delta \quad \text { for all } n \geq N_{0} .
$$

We assert that for any fixed $k \geq N_{0}$,

$$
G\left(x_{k}, x_{k+r}, x_{k+r}\right) \leq \varepsilon \quad \text { for all } r=1,2, \ldots
$$

holds. To prove the assertion, we use the method of induction. Regarding (2.9), the assertion (2.10) is satisfied for $r=1$. Suppose the assertion (2.10) is satisfied for $r=1,2, \ldots, m$ for some $m \in \mathbb{N}$. For $r=m+1$, by the help of (G5) and (2.9), we consider

$$
\begin{aligned}
G\left(x_{k-1}, x_{k+m}, x_{k+m}\right) & \leq G\left(x_{k-1}, x_{k}, x_{k}\right)+G\left(x_{k}, x_{k+m}, x_{k+m}\right) \\
& <\varepsilon+\delta .
\end{aligned}
$$

If $G\left(x_{k-1}, x_{k+m}, x_{k+m}\right) \geq \varepsilon$, then by $(1.8)$ we get

$$
G\left(x_{k}, x_{k+m+1}, x_{k+m+1}\right)=G\left(T x_{k-1}, T x_{k+m}, T x_{k+m}\right)<\varepsilon .
$$

Hence (2.10) is satisfied.

If $G\left(x_{k-1}, x_{k+m}, x_{k+m}\right)=0$, then by (G2), we derive that $x_{k-1}=x_{k+m}$ and hence $x_{k}=T x_{k-1}=$ $T x_{k+m}=x_{k+m+1}$. By (G1), we have

$$
G\left(x_{k}, x_{k+m+1}, x_{k+m+1}\right)=G\left(x_{k}, x_{k}, x_{k}\right)=0<\varepsilon,
$$

and thus (2.10) is satisfied.

If $0<G\left(x_{k-1}, x_{k+m}, x_{k+m}\right)<\varepsilon$, then by Remark 18,

$$
G\left(x_{k}, x_{k+m+1}, x_{k+m+1}\right)=G\left(T x_{k-1}, T x_{k+m}, T x_{k+m}\right) \leq G\left(x_{k-1}, x_{k+m}, x_{k+m}\right)<\varepsilon .
$$

Consequently, (2.10) is satisfied for $r=m+1$. Hence, $G\left(x_{k}, x_{k+r}, x_{k+r}\right) \leq \varepsilon$ for all $k \geq N_{0}$ and $r \geq 1$, which means

$$
G\left(x_{n}, x_{m}, x_{m}\right)<\varepsilon \quad \forall m \geq n \geq N_{0} .
$$

Then, for all $n \geq m \geq N_{0}$, by (2.13), we have

$$
G\left(x_{n}, x_{m}, x_{m}\right)=G\left(x_{m}, x_{n}, x_{m}\right) \leq G\left(x_{m}, x_{n}, x_{n}\right)+G\left(x_{n}, x_{n}, x_{m}\right)=2 G\left(x_{m}, x_{n}, x_{n}\right)<2 \varepsilon
$$

Thus, for all $m, n \geq N_{0}$, there holds

$$
G\left(x_{n}, x_{m}, x_{m}\right)<2 \varepsilon .
$$


By Proposition $6,\left\{x_{n}\right\}$ is a G-Cauchy sequence. Since $(X, G)$ is $G$-complete, there exists $u \in X$ such that

$$
\lim _{n \rightarrow \infty} G\left(x_{n}, u, u\right)=0
$$

We will show now that $u \in X$ is a fixed point of $T$, that is, $u=T u$. Since $T$ is $G$ continuous, the sequence $\left\{T x_{n}\right\}=\left\{x_{n+1}\right\}$ converges to $T u$, that is,

$$
\lim _{n \rightarrow \infty} G\left(T x_{n}, T x_{n}, T u\right)=\lim _{n \rightarrow \infty} G\left(T x_{n}, T u, T u\right)=0 .
$$

On the other hand, the rectangle inequality (G5) yields that

$$
\begin{aligned}
G(u, T u, T u) & \leq G\left(u, x_{n+1}, x_{n+1}\right)+G\left(x_{n+1}, T u, T u\right) \\
& =G\left(u, x_{n+1}, x_{n+1}\right)+G\left(T x_{n}, T u, T u\right) .
\end{aligned}
$$

Letting $n \rightarrow \infty$ in (2.16), we conclude that $G(u, T u, T u)=0$. Hence, $u=T u$, that is, $u \in$ is a fixed point of $T$.

To prove the uniqueness, we assume that $v \in X$ is another fixed point of $T$. By the assumptions, we know that there exists $w \in X$ such that $u \preceq w$ and $v \preceq w$. By Remark 18, we get

$$
G(u, T w, T w)=G(T u, T w, T w) \leq G(u, w, w)
$$

Since $T$ is nondecreasing, $T u \preceq T w$. Again by Remark 18 , we get

$$
G\left(u, T^{2} w, T^{2} w\right)=G(T(T u), T(T w), T(T w)) \leq G(T u, T w, T w)=G(u, T w, T w)
$$

Continuing in this way, we conclude

$$
G\left(u, T^{n} w, T^{n} w\right) \leq \cdots \leq G(u, T w, T w) \leq G(u, w, w)
$$

Let $s_{n}=G\left(u, T^{n} w, T^{n} w\right)$. Hence, we conclude that $\left\{s_{n}\right\}$ is a non-increasing sequence bounded below by zero. Thus, there exists $L \geq 0$ such that

$$
\lim _{n \rightarrow \infty} s_{n}=L=\inf _{n} s_{n}
$$

We claim that $L=0$. Suppose, on the contrary, that $L>0$. Choose $\varepsilon=L$ and $\delta>0$ be such that (1.8) holds. Then, there exists $n_{0}$ such that $L \leq G\left(u, T^{n_{0}} w, T^{n_{0}} w\right)<L+\delta$, which implies

$$
G\left(u, T^{n_{0}+1} w, T^{n_{0}+1} w\right)=G\left(T u, T^{n_{0}+1} w, T^{n_{0}+1} w\right)<L .
$$

This contradicts with the definition of $L$. Hence,

$$
\lim _{n \rightarrow \infty} G\left(u, T^{n} w, T^{n} w\right)=0
$$


Similarly, one can also obtain

$$
\lim _{n \rightarrow \infty} G\left(v, T^{n} w, T^{n} w\right)=0
$$

In view of (2.17), (2.18) and

$$
G(u, v, v) \leq G\left(u, T^{n} w, T^{n} w\right)+G\left(T^{n} w, v, v\right)
$$

we deduce $G(u, v, v)=0$, i.e., $u=v$. Hence, the fixed point of $T$ is unique.

Corollary 20 Let $(X, \preceq)$ be a partially ordered set endowed with a G-metric and $T: X \rightarrow X$ be a given mapping. Suppose that the following conditions hold:

(i) $(X, G)$ is G-complete;

(ii) $T$ is nondecreasing (with respect to $\preceq$ );

(iii) there exists $x_{0} \in X$ such that $x_{0} \preceq T x_{0}$;

(iv) $T$ is G-continuous;

(v) $T: X \rightarrow X$ is G-Meir-Keeler contractive.

Then $T$ has a fixed point. Moreover, if for all $(x, y) \in X \times X$, there exists $w \in X$ such that $x \preceq w$ and $y \preceq w$, we obtain the uniqueness of the fixed point.

Substituting the condition (iv) in Theorem 19 by the condition that $X$ is ordered complete, we can get the following result.

Theorem 21 Let $(X, \preceq)$ be a partially ordered set endowed with a G-metric and $T: X \rightarrow X$ be a given mapping. Suppose that the following conditions hold:

(i) $(X, G)$ is G-complete;

(ii) $T$ is nondecreasing (with respect to $\preceq$ );

(iii) there exists $x_{0} \in X$ such that $x_{0} \preceq T x_{0}$;

(iv) $X$ is ordered complete;

(v) $T: X \rightarrow X$ is G-Meir-Keeler contractive of second type.

Then $T$ has a fixed point. Moreover, if for all $(x, y) \in X \times X$ there exists $w \in X$ such that $x \preceq w$ and $y \preceq w$, we obtain the uniqueness of the fixed point.

Proof Let $x_{n}$ and $u$ be as in the proof of Theorem 19. We only need to show $u=T u$. Since $X$ is ordered complete, in view of (2.2) and (2.14), we conclude $x_{n} \preceq u$ for all $n$. Then, by Remark 18, (G5) and (2.14), we get

$$
\begin{aligned}
G(T u, u, u) & \leq G\left(T u, x_{n}, x_{n}\right)+G\left(x_{n}, u, u\right) \\
& =G\left(T x_{n-1}, T x_{n-1}, T u\right)+G\left(x_{n}, u, u\right) \\
& \leq G\left(x_{n-1}, x_{n-1}, u\right)+G\left(x_{n}, u, u\right) .
\end{aligned}
$$

Letting $n \rightarrow \infty$, we conclude $G(T u, u, u)=0$, i.e., $T u=u$.

Corollary 22 Let $(X, \preceq)$ be a partially ordered set endowed with a G-metric and $T: X \rightarrow X$ be a given mapping. Suppose that the following conditions hold:

(i) $(X, G)$ is G-complete;

(ii) $T$ is nondecreasing (with respect to $\preceq$ ); 
(iii) there exists $x_{0} \in X$ such that $x_{0} \preceq T x_{0}$;

(iv) $X$ is ordered complete;

(v) $T: X \rightarrow X$ is G-Meir-Keeler contractive.

Then $T$ has a fixed point. Moreover, if for all $(x, y) \in X \times X$ there exists $w \in X$ such that $x \preceq w$ and $y \preceq w$, we obtain the uniqueness of the fixed point.

Theorem 23 Let $(X, \preceq)$ be a partially ordered set endowed with a G-metric and $T: X \rightarrow X$ be a given mapping. Suppose that there exists a function $\varphi:[0, \infty) \rightarrow[0, \infty)$ satisfying the following conditions

(F1) $\varphi(0)=0$ and $\varphi(t)>0$ for all $t>0$;

(F2) $\varphi$ is nondecreasing and right continuous;

(F3) for every $\varepsilon>0$, there exists $\delta$ such that

$$
\varepsilon \leq \varphi(G(x, y, y))<\varepsilon+\delta \quad \text { implies } \quad \varphi(G(T x, T y, T y))<\varphi(\varepsilon)
$$

for all $(x, y) \in X \times X$ with $x \preceq y$. Then $T$ is G-Meir-Keeler contractive of second type.

Proof We take $\varepsilon>0$. Due to (F1), we have $\varphi(\varepsilon)>0$. Thus there exists $\theta>0$ such that

$$
\varphi(\varepsilon) \leq \varphi(G(x, y, y))<\varphi(\varepsilon)+\theta \quad \text { implies } \quad \varphi(G(T x, T y, T y))<\varphi(\varepsilon) .
$$

From the right continuity of $\varphi$, there exists $\delta>0$ such that $\varphi(\varepsilon+\delta)<\varphi(\varepsilon)+\theta$. Fix $(x, y) \in$ $X \times X$ with $x \preceq y$ such that $\varepsilon \leq G(x, y, y)<\varepsilon+\delta$. So, we have

$$
\varphi(\varepsilon) \leq \varphi(G(x, y, y)) \leq \varphi(\varepsilon+\delta)<\varphi(\varepsilon)+\theta .
$$

Hence, $\varphi(G(T x, T y, T y))<\varphi(\varepsilon)$. Thus, we have $G(T x, T y, T y)<\varepsilon$, which completes the proof.

Since a function $t \rightarrow \int_{0}^{t} f(s) d s$ is absolutely continuous, we derive the following corollary from Theorem 23 and Theorem 19.

Corollary 24 Let $(X, \preceq)$ be a partially ordered set endowed with a G-metric, $T: X \rightarrow X$ be a given mapping, and $f$ be a locally integrable function from $[0, \infty)$ into itself satisfying $\int_{0}^{t} f(s) d s>0$ for all $t>0$. Assume that the conditions (i)-(iv) of Theorem 19 hold, and for each $\varepsilon>0$, there exists $\delta>0$ such that

$$
\varepsilon \leq \int_{0}^{G(x, y, y)} f(s) d s<\varepsilon+\delta \Rightarrow \int_{0}^{G(T x, T y, T y)} f(s) d s<\int_{0}^{\varepsilon} f(s) d s
$$

for all $x, y \in X$ with $x \leq y$. Then $T$ has a fixed point. Moreover, iffor all $(x, y) \in X \times X$, there exists $w \in X$ such that $x \preceq w$ and $y \preceq w$, we obtain the uniqueness of the fixed point.

The authors declare that they have no competing interests. 


\section{Author details}

'College of Mathematics and Information Science, Jiangxi Normal University, Nanchang, Jiangxi 330022, People's Republic of China. ${ }^{2}$ Department of Mathematics, Atilim University, İncek, Ankara 06836, Turkey.

\section{Acknowledgements}

The authors thank the referees for their valuable comments that helped to improve the text. Hui-Sheng Ding acknowledges support from the NSF of China, and the Program for Cultivating Youths of Outstanding Ability in Jiangxi Normal University.

Received: 26 August 2012 Accepted: 6 February 2013 Published: 22 February 2013

\section{References}

1. Abbas, M, Khan, AR, Nazir, T: Coupled common fixed point results in two generalized metric spaces. Appl. Math. Comput. 217, 6328-6336 (2011)

2. Aydi, $\mathrm{H}$, Postolache, $\mathrm{M}$, Shatanawi, $\mathrm{W}$ : Coupled fixed point results for $(\psi, \phi)$-weakly contractive mappings in ordered G-metric spaces. Comput. Math. Appl. (2012). doi:10.1016/j.camwa.2011.11.022

3. Aydi, H, Damjanović, B, Samet, B, Shatanawi, W: Coupled fixed point theorems for nonlinear contractions in partially ordered G-metric spaces. Math. Comput. Model. 54, 2443-2450 (2011)

4. Banach, S: Sur les opérations dans les ensembles abstraits et leur application aux équations intégrales. Fundam. Math. 3, 133-181 (1922)

5. Choudhury, BS, Maity, P: Coupled coincidence point result in generalized metric spaces. Math. Comput. Model. 54, 73-79 (2011)

6. Ding, H-S, Karapınar, E: A note on some coupled fixed point theorems on G-metric space. J. Inequal. Appl. 2012, 170 (2012)

7. Harjani, J, López, B, Sadarangani, K: A fixed point theorem for Meir-Keeler contractions in ordered metric spaces. Fixed Point Theory Appl. 2011, 83 (2011)

8. Jachymski, J: Equivalent conditions for generalized contractions on (ordered) metric spaces. Nonlinear Anal. 74 768-774 (2011)

9. Jachymski, J: Equivalent conditions and the Meir-Keeler type theorems. J. Math. Anal. Appl. 194, $293-303$ (1995)

10. Kadelburg, Z, Radenović, S: Meir-Keeler-type conditions in abstract metric spaces. Appl. Math. Lett. 24, 1411-1414 (2011)

11. Karapınar, E, Erhan, M, Ulus, AY: Cyclic contractions on G-metric spaces. Abstr. Appl. Anal. 2012, Article ID 182947 (2012)

12. Khan, SM, Swaleh, M, Sessa, S: Fixed points theorems by altering distances between the points. Bull. Aust. Math. Soc. 30, 1-9 (1984)

13. Meir, A, Keeler, E: A theorem on contraction mapping. J. Math. Anal. Appl. 28, 326-329 (1969)

14. Mustafa, Z: A new structure for generalized metric spaces with applications to fixed point theory. PhD thesis, The University of Newcastle, Australia (2005)

15. Mustafa, Z, Sims, B: A new approach to generalized metric spaces. J. Nonlinear Convex Anal. 7, $289-297$ (2006)

16. Mustafa, Z, Obiedat, H, Awawdeh, F: Some fixed point theorem for mapping on complete G-metric spaces. Fixed Point Theory Appl. 2008, Article ID 189870 (2008)

17. Mustafa, Z, Khandaqji, M, Shatanawi, W: Fixed point results on complete G-metric spaces. Studia Sci. Math. Hung. 48, 304-319 (2011)

18. Mustafa, Z, Sims, B: Fixed point theorems for contractive mappings in complete G-metric spaces. Fixed Point Theory Appl. 2009, Article ID 917175 (2009)

19. Mustafa, Z, Shatanawi, W, Bataineh, M: Existence of fixed point results in G-metric spaces. Int. J. Math. Math. Sci. 2009, Article ID 283028 (2009)

20. Nieto, JJ, Rodríguez-López, R: Contractive mapping theorems in partially ordered sets and applications to ordinary differential equations. Order 22, 223-239 (2005)

21. Luong, NV, Thuan, NX: Coupled fixed point theorems in partially ordered G-metric spaces. Math. Comput. Model. 55, 1601-1609 (2012)

22. Ran, ACM, Reurings, MCB: A fixed point theorem in partially ordered sets and some applications to matrix equations. Proc. Am. Math. Soc. 132, 1435-1443 (2004)

23. Samet, B: Coupled fixed point theorems for a generalized Meir-Keeler contraction in partially ordered metric spaces. Nonlinear Anal. 72, 4508-4517 (2010)

24. Shatanawi, W: Coupled fixed point theorems in generalized metric spaces. Hacet. J. Math. Stat. 40, 441-447 (2011)

25. Suzuki, T: Fixed-point theorem for asymptotic contractions of Meir-Keeler type in complete metric spaces. Nonlinear Anal. 64, 971-978 (2006)

26. Agarwal, RP, Karapınar, E: Remarks on some coupled fixed point theorems in G-metric spaces. Fixed Point Theory Appl. 2013, 2 (2013)

doi:10.1186/1687-1812-2013-35

Cite this article as: Ding and Karapınar: Meir-Keeler type contractions in partially ordered G-metric spaces. Fixed Point Theory and Applications 2013 2013:35. 